\title{
Diagnóstico laboratorial da esporotricose felina em amostras coletadas no estado do Rio de Janeiro, Brasil: limitações da citopatologia por imprint
}

\author{
Laboratory diagnosis of feline sporotrichosis in samples from Rio de Janeiro State, \\ Brazil: imprint cytopathology limitations
}

Pãmella Antunes de Macêdo-Sales, Simone Rocha Leal da Silveira Souto, Carolina Airão Destefani, Ricardo Pereira de Lucena, Elisabeth Martins da Silva da Rocha, Andréa Regina de Souza Baptista

Universidade Federal Fluminense, Instituto Biomédico, Departamento de Microbiologia e Parasitologia, Laboratório de Micologia Médica e Molecular, Niterói, Rio de Janeiro, Brasil

\begin{abstract}
RESUMO
INTRODUÇÃO: O estado do Rio de Janeiro vem passando por uma epidemia de esporotricose, considerada doença negligenciada, de transmissão zoonótica pelo felino doméstico (Felis catus). Apesar do isolamento de fungos do complexo Sporothrix schenckii em cultura micológica ser o padrão-ouro no diagnóstico, a citopatologia por imprint das lesões é rotineiramente utilizada na prática da clínica veterinária. OBJETIVO: Avaliar o desempenho da citopatologia como método diagnóstico da esporotricose em gatos com suspeita dessa micose, provenientes de diferentes áreas geográficas do estado do Rio de Janeiro, Brasil. MATERIAIS E MÉTODOS: Após avaliação clínica, o swab do exsudato e lâminas via imprint da lesão foram coletados de 196 felinos domésticos, com posterior semeadura em Ágar Sabouraud Dextrose e Mycosel e coloração das lâminas por Panótico Rápido. RESULTADOS: Dos 196 animais, 102 (52,0\%) foram diagnosticados com esporotricose. Para 50,0\% da população, os resultados da cultura e da citopatologia foram discordantes ( $\left.p<0,0001 ; \chi^{2}=32,960\right)$, com a cultura micológica mais sensível $(95,2 \%)$ que a citopatologia $(52,6 \%)$. Para os animais tratados com dosagem de itraconazol $\geq 100 \mathrm{mg} /$ dia, foi observada uma significante redução no desempenho diagnóstico da citopatologia $(p=0,0136)$. Contudo, o mesmo não foi verificado em relação ao tempo de tratamento ou às demais dosagens $(p>0,05)$. CONCLUSÃO: A dosagem de itraconazol $\geq 100 \mathrm{mg} /$ dia, independente da duração do tratamento, reduz a sensibilidade diagnóstica da citopatologia. Tal resultado representa um alerta para o uso dessa ferramenta como recurso único para a confirmação diagnóstica da esporotricose felina na rotina da clínica veterinária.
\end{abstract}

Palavras-chave: Zoonoses; Sporothrix; Gatos; Técnicas e Procedimentos Diagnósticos.

\begin{abstract}
INTRODUCTION: Rio de Janeiro State is undergoing an epidemic of sporotrichosis, a neglected disease of zoonotic transmission by domestic cats (Felis catus). The isolation of the fungal of Sporothrix schenckii complex in mycological culture is the gold standard for sporotrichosis diagnosis; however, imprint cytopathology of lesions is routinely used in veterinary clinical practice. OBJECTIVE: To evaluate the performance of cytopathology as a diagnostic method for sporotrichosis in a population of cats with suspicion of this mycosis, from different geographic areas of Rio de Janeiro State, Brazil. MATERIALS AND METHODS: After clinical evaluation, exudate swab and slides by imprint of the lesion were collected from 196 domestic cats, with subsequent sowing in Sabouraud Dextrose and Mycosel Agar and slides staining by quick panoptic stain. RESULTS: Of the 196 animals, 102 (52.0\%) were diagnosed with sporotrichosis. The results of culture and cytopathology were discordant $\left(p<0.0001 ; \chi^{2}=32.960\right)$, with mycological culture (95.2\%) more sensitive than cytopathology (52.6\%). For animals treated with itraconazole dosage $\geq 100 \mathrm{mg} /$ day, a significant reduction in the diagnostic performance of cytopathology was observed $(p=0.0136)$. However, the same was not verified in relation to treatment time or other dosages $(p>0.05)$. CONCLUSION: Regardless of the duration of treatment, the dose of itraconazole $\geq 100 \mathrm{mg} /$ day reduces the diagnostic sensitivity of cytopathology. This result is an alert for the use of this method as a unique resource for the diagnostic confirmation of feline sporotrichosis in veterinary clinical routine.
\end{abstract}

Keywords: Zoonoses; Sporothrix; Gatos; Técnicas e Procedimentos Diagnósticos.

\section{Correspondência / Correspondence:}

Andréa Regina de Souza Baptista

Universidade Federal Fluminense, Instituto Biomédico, Departamento de Microbiologia e Parasitologia

Rua Prof. Hernani Pires de Melo, 101. Bairro: São Domingos. CEP: 24210-130 -Niterói, Rio de Janeiro. Brasil - Tel.: +55 (21) $2629-2439$

E-mail: andrearegina@id.uff.br 


\section{INTRODUÇÃO}

A esporotricose é uma micose que ocorre após a implantação traumática de fungos do clado patogênico do complexo Sporothrix schenckii. Recentemente, foi proposta a existência de pelo menos seis espécies patogênicas no complexo, com especial valor ao Sporothrix brasiliensis, exclusivamente isolado em território brasileiro e principal agente do cenário epidêmico instalado no estado do Rio de Janeiro ${ }^{1,2}$. Os fungos desse complexo são dimórficos, de distribuição cosmopolita e frequentemente isolados em locais de clima quente e úmido, a partir do solo e da matéria orgânica vegetal, como cascas de árvores e espinhos, além de material em decomposição. A via clássica de transmissão está relacionada a traumas cutâneos com inoculação de matéria orgânica ${ }^{3,4,5}$.

Desde a década de 1990, a esporotricose vem sendo descrita como uma das mais importantes micoses de implantação traumática, tendo apresentado significante mudança no perfil de transmissão, assumindo o status de epidemia no Rio de Janeiro. Apesar disso, segue subnotificada nesse Estado, figurando entre as doenças negligenciadas. Nesse contexto, o felino doméstico (Felis catus) é o principal animal acometido por essa micose, bem como transmissor, representando papel central na forma de transmissão zoonótica ${ }^{4,6}$. Assim, os felinos domésticos com esporotricose promovem a implantação traumática do fungo no tecido subcutâneo humano e de outros animais e também apresentam manifestação clínica exuberante dessa micose, na maioria dos casos cutânea disseminada. Dado o hábito felino de arranhar troncos de árvores e de outras superfícies e o de enterrar suas fezes, aliados ao de lambedura de seu corpo, as unhas e a nasofaringe/cavidade oral podem abrigar estruturas desse fungo, expondo o animal à infecção, assim como os demais hospedeiros ${ }^{7,8}$. Os animais doentes, por sua vez, ampliam a contaminação ambiental, sendo preocupante o descarte inapropriado dos cadáveres, prática comumente observada?. Além disso, os Felis catus machos têm por comportamento as brigas por território e também por fêmeas, tornando, assim, os animais em idade reprodutiva ainda mais expostos $^{5,10,11}$. Atualmente, essa dermatozoonose tem sido reportada em outros estados brasileiros, como Minas Gerais, São Paulo, Paraná e Rio Grande do Sul2,11,12,13,14; entretanto, somente no Rio de Janeiro sua notificação é compulsória.

Os procedimentos laboratoriais para o diagnóstico da esporotricose já estão bem estabelecidos, e o isolamento em cultura dos fungos do complexo S. schenckii constitui o padrão-ouro, que deve ser seguido da identificação macro e micromorfológicas e da prova de termoconversão in vitro. Na prática clínica veterinária, no entanto, o diagnóstico citopatológico é utilizado rotineiramente, elaborado a partir do imprint das lesões de gatos domésticos em lâmina de vidro, corado pelo Panótico Rápido (bateria de corantes em que há predominância de tons vermelhos, $\mathrm{pH}$ ácido e azulados, $\mathrm{pH}$ básico, que cora as células em $1 \mathrm{~min}$ ), evidenciando estruturas leveduriformes abundantes, internalizadas ou não, nos fagócitos ${ }^{5,7,15}$. Apesar desse segundo método representar uma alternativa diagnóstica rápida, de fácil execução e de baixo custo, sendo, portanto, frequentemente empregado na rotina clínica veterinária, é considerado presuntivo. A cultura micológica, por sua vez, apresenta limitações relacionadas à impossibilidade de processamento do espécime clínico no local do atendimento ambulatorial, custo mais elevado e o maior tempo para a liberação dos resultados (cerca de 20 dias) ${ }^{3}$.

Atualmente, a esporotricose permanece acometendo ambos os hospedeiros, humanos e felinos, com números crescentes, preocupantes, em território fluminense e em outros estados do Brasil, como Minas Gerais, Espírito Santo, São Paulo, Paraná e Rio Grande do Sul. Nesse contexto, um ponto relevante reside no fato de que estratégias futuras de prevenção e controle da esporotricose zoonótica dependem do diagnóstico rápido da doença no felino doméstico, etapa tida como crucial para o tratamento eficaz. Tal importância baseia-se na interrupção da transmissão entre felinos doentes e também desses para seus tutores e familiares. Assim, o presente estudo teve por objetivo avaliar o desempenho da citopatologia como método diagnóstico da esporotricose em uma população de gatos com suspeita dessa micose, provenientes de diferentes áreas geográficas do Rio de Janeiro.

\section{MATERIAIS E MÉTODOS}

A presente pesquisa é um estudo transversal, aprovado pelo Comitê de Ética no Uso de Animais da Universidade Federal Fluminense (CEUA-UFF, protocolo número 208/2012 em 13 de dezembro de 2012). As áreas geográficas abrangidas foram as Zonas Oeste, Centro-Sul e Norte do município do Rio de Janeiro (três clínicas veterinárias e um ambulatório), a Baixada Fluminense (Região Metropolitana I, incluindo os municípios de Duque de Caxias, Nilópolis, São João de Meriti e Magé - uma clínica veterinária), a Grande Niterói (Região Metropolitana II, incluindo os municípios de Niterói, São Gonçalo e Itaboraí quatro clínicas veterinárias e um ambulatório), a Região Serrana (Petrópolis, Teresópolis e Cachoeira de Macacu - uma clínica veterinária e um polo de cooperação) e a Baixada Litorânea (Arraial do Cabo, Araruama, Cabo Frio, Búzios, Maricá, São Pedro da Aldeia e Saquarema - duas clínicas veterinárias e dois polos de cooperação), como descrito pela Secretaria de Saúde do Rio de Janeiro (SES/RJ).

Durante 12 meses (janeiro de 2013 a janeiro de 2014), diferentes ambulatórios, clínicas veterinárias e polos de cooperação receberam, via demanda passiva ou após busca ativa, animais que foram atendidos e submetidos à avaliação clínica por médicos veterinários. Desse modo, um total de 196 felinos domésticos com lesões clínicas consideradas 
sugestivas da esporotricose foi incluído. As informações referentes aos dados clínico-epidemiológicos, tais como procedência, castração, localização e características das lesões, comorbidades, contato com outros animais com lesões de pele ou não, com o solo e plantas ou não, entre outras, foram registradas em um formulário padronizado. Todos os tutores, cuidadores, médicos veterinários, ou ainda aqueles que se responsabilizaram por animais abandonados ou que não possuíam tutor/proprietário, foram esclarecidos quanto aos objetivos e métodos do estudo anteriormente à coleta das amostras e assinaram um termo de consentimento livre e esclarecido. $\bigcirc$ critério de inclusão foi a presença de lesões cutâneas suspeitas de esporotricose, sem limitações quanto à idade, ao sexo, à raça, ao estado geral de saúde (doenças crônicas ou infecciosas e estado nutricional), em tratamento ou não com antifúngicos, segundo atestado pelos médicos veterinários colaboradores.

Após a avaliação clínica, a citopatologia por imprint foi efetuada por meio da pressão suave de lâmina de vidro sobre a lesão suspeita, previamente limpa com solução fisiológica estéril, caso houve crostas, seguida do acondicionamento em tubo porta-lâminas para posterior coloração. Cada felino, em razão do número das lesões suspeitas, teve de uma a três lâminas coletadas. As lâminas com o material colhido foram fixadas em álcool absoluto $(99,5 \%)$ e coradas pelo método do Panótico Rápido (LABORCLIN, Pinhais, Paraná, Brasil), baseado no princípio estabelecido por Romanowsky ${ }^{16}$. Após a coloração, as lâminas foram secas em temperatura ambiente e, em seguida, efetuada a análise em microscopia óptica $(400 X)$ por, pelo menos, dois pesquisadores de forma independente. Foram consideradas positivas aquelas com estruturas compatíveis com as leveduras do complexo S. schenckii, internalizadas ou não nos fagócitos, independente de sua quantidade. Foram consideradas negativas as lâminas que não apresentaram as referidas estruturas após varredura completa de todos os campos da lâmina corada.

A fim de efetuar a cultura micológica, o exsudato das lesões foi coletado com o auxílio de um swab estéril, posteriormente acondicionado em tubo contendo $1 \mathrm{~mL}$ de solução salina estéril (0,9\%) e imediatamente encaminhado ao Laboratório de Micologia Médica e Molecular do Instituto Biomédico da Universidade Federal Fluminense, em Niterói, para processamento. $\bigcirc$ swab foi então utilizado para a semeadura em dois tubos: um contendo Ágar Sabouraud Dextrose 2\% (BD, New Jersey, EUA) e o outro, Mycose ${ }^{\circledR}$ (BD, New Jersey, EUA). Foram consideradas colônias sugestivas aquelas membranosas, achatadas, pregueadas e raramente apresentando um micélio aéreo cinza nas bordas. Por fim, as provas do dimorfismo térmico, via crescimento da forma de levedura em ágar Brain Heart Infusion (BD, New Jersey, EUA), e do microcultivo em Ágar Batata Dextrose (BD, New
Jersey, EUA) foram realizadas para confirmação diagnóstica.

Os dados obtidos nos formulários padronizados foram armazenados em planilha no software Excel ${ }^{\circledR}$ 2010 (Microsoft, Washington, EUA). Para avaliação do grau de discordância entre os métodos laboratoriais empregados, foi aplicado o teste de McNemar. Os parâmetros sensibilidade, especificidade, acurácia, valor preditivo negativo, valor preditivo positivo e falso-negativo foram determinados pelo screening test, todos efetuados por meio do software BioEstat v5.3 (Instituto Mamirauá, Amazonas, Brasil). Foi ainda investigada a possível influência de outros fatores no desempenho diagnóstico da citopatologia e cultura, tais como: aspecto das lesões cutâneas (presença de crostas, pus ou sangue, eritema, úlceras, nódulos); sinais clínicos (dificuldade respiratória, espirros, secreção nasal, emagrecimento, apatia, epistaxe, secreção ocular, dificuldade de locomoção); e vigência, dosagem e duração do tratamento antifúngico. Para todos os testes, o nível de significância adotado foi de $\alpha<0,05$.

\section{RESULTADOS}

Dentre os animais assistidos, $102 \quad(52,0 \%)$ apresentaram cultura micológica positiva e/ou citopatologia por imprint sugestiva do complexo S. schenckii e foram diagnosticados como portadores da doença. Nos animais com essa micose, a maioria (54; 52,9\%) era da Zona Norte da capital, seguida por $17(16,7 \%)$ da Zona Oeste, 13 (12,7\%) da Grande Niterói, sete $(6,9 \%)$ da Região Serrana, cinco $(4,9 \%)$ da Baixada Litorânea, quatro (3,9\%) da Baixada Fluminense e dois (2,0\%) da Zona Centro-Sul da capital.

A população felina acometida por essa zoonose apresentou idade média de $3,75 \pm 3,1$ anos e era composta, em sua maioria, por machos (62; 60,8\%), não castrados $(57 ; 55,9 \%)$, sem raça definida (90; 88,2\%). Os aspectos mais frequentes das lesões foram: ulceração $(76 ; 74,5 \%)$, presença de sangue (70; 68,6\%) e crostas (60; 58,8\%). A região mais acometida pelas lesões foi a cabeça $(57 ; 55,9 \%)$, seguida pelos membros pélvicos $(27 ; 26,5 \%)$ e torácicos (26; 25,5\%). Mais de um terço deles $(42 ; 41,2 \%)$ estava em vigência de tratamento com o itraconazol, via oral, em diferentes dosagens (25 mg a $100 \mathrm{mg}$ ) e vigências (um dia a cinco anos) no momento da coleta dos espécimes clínicos. Não houve diferença estatística entre os parâmetros acima descritos, quando comparados aos animais provenientes de diferentes áreas geográficas do Rio de Janeiro.

Quando comparados os resultados da citopatologia por imprint com os da cultura micológica, houve resultados discordantes: dos 56 com citopatologia positiva, 51 (91,1\%) apresentaram cultura positiva e cinco (8,9\%) negativa; dos 140 com citopatologia negativa, 46 (32,9\%) tiveram cultura positiva e 94 $(67,1 \%)$ negativa (Tabela 1$)$. 
Tabela 1 - Combinações entre os resultados verificados pelos métodos de citopatologia da lesão e cultura micológica do exsudato da lesão nos 196 felinos com suspeita de esporotricose, no estado do Rio de Janeiro, Brasil, 2013-2014

\begin{tabular}{|c|c|c|c|c|c|c|}
\hline \multirow{2}{*}{ Métodos } & \multicolumn{2}{|c|}{ Cultura positiva } & \multicolumn{2}{|c|}{ Cultura negativa } & \multicolumn{2}{|c|}{ Total } \\
\hline & $N$ & $\%$ & $N$ & $\%$ & $N$ & $\%$ \\
\hline Citopatologia positiva & 51 & 91,1 & 5 & 8,9 & 56 & 28,6 \\
\hline Citopatologia negativa & 46 & 32,9 & 94 & 67,1 & 140 & 71,4 \\
\hline Total & 97 & 49,5 & 99 & 50,5 & 196 & 100,0 \\
\hline
\end{tabular}

Utilizando a cultura micológica do exsudato da lesão como padrão-ouro, foram efetuados os cálculos dos parâmetros de sensibilidade, especificidade, acurácia, falso-negativo e dos valores preditivos positivo e negativo frente à citopatologia por imprint (Tabela 2). $\bigcirc$ teste de maiores sensibilidade e especificidade foi a cultura micológica com (95,2\% e $100,0 \%$, respectivamente); enquanto a citopatologia por imprint alcançou sensibilidade de $52,6 \%$ e especificidade de 95,0\%. A cultura micológica foi também mais acurada do que a citopatologia por imprint e a porcentagem de falso-negativos do primeiro teste foi muito inferior ao segundo (4,8\% e $47,4 \%)$. A citopatologia por imprint teve valor preditivo positivo menor $(91,1 \%)$ do que a cultura $(100,0 \%)$, enquanto os valores preditivos negativos foram diferentes: cultura - 94,7\%; citopatologia - 67,1\%. Finalmente, com o objetivo de avaliar o grau de discordância entre os testes, foi calculado o p-valor para os pares discordantes entre o diagnóstico por cultura micológica e por citopatologia por imprint a partir das lesões. A cultura e a citopatologia produziram respostas discordantes ( $p<0,0001 ; \chi^{2}=32,96078$ ) (Tabela 2).

Tabela 2 - Percentual dos parâmetros analíticos e o teste de McNemar para cultura e para citopatologia nos diagnósticos de esporotricose em felinos do estado do Rio de Janeiro, Brasil, 2013-2014

\begin{tabular}{l|c}
\hline \multicolumn{1}{c|}{ Parâmetros } & Citopatologia por imprint (\%) \\
\hline Sensibilidade & 52,6 \\
\hline Especificidade & 95,0 \\
\hline Acurácia & 74,0 \\
\hline Falso-negativo & 47,4 \\
\hline VPP & 91,1 \\
\hline VPN & 67,1 \\
\hline Teste de McNemar & $p<0,0001 ; \chi^{2}=31,3725$ \\
Índice de Kappa & 0,48 \\
\hline
\end{tabular}

VPP: Valor preditivo positivo; VPN: Valor preditivo negativo.
Foram investigadas a possível influência dos aspectos clínicos e da gravidade das lesões cutâneas, bem como a presença de sinais coadjuvantes no desempenho diagnóstico da citopatologia e da cultura, e, dentre essas variáveis, nenhuma interferiu no desempenho de ambos os métodos $(p>0,05)$. Por outro lado, o tratamento prévio com antifúngicos mostrou interferência na citopatologia, visto que, para os animais tratados com itraconazol em dosagens iguais ou superiores a $100 \mathrm{mg} /$ dia, foi observada significante redução na sensibilidade diagnóstica da citopatologia ( $p=0,0136$ ). Contudo, o mesmo não foi verificado em relação ao tempo de tratamento $(p>0,05)$.

\section{DISCUSSÃO}

Clinicamente, as manifestações da esporotricose felina podem ser confundidas com outras doenças infectoparasitárias, tais como a criptococose, a leishmaniose e outras afecções cutâneas, como atopia e, até mesmo, neoplasias, enfatizando a necessidade do diagnóstico laboratorial. Apesar das técnicas diagnósticas para a esporotricose felina estarem bem estabelecidas, pouco se sabe sobre a possível interferência de fatores externos ao método, como características da lesão, ocorrência de comorbidades ou mesmo a vigência de tratamento no desempenho da citopatologia por imprint e da cultura micológica ${ }^{3,11}$.

No presente estudo, a idade média dos felinos com esporotricose foi de 3,71 anos e a maioria foi composta por machos não castrados e sem raça definida. Realmente, nessa idade, os gatos iá atingiram a maturidade sexual, quando ocorre o cio, aumentando a chance de brigas pelas fêmeas e a consequente exposição aos animais portadores da doença. Esses achados corroboram relatos anteriores que descrevem que gatos machos e não castrados apresentam maior risco de exposição ao agente da esporotricose e, em consequência, adoecem mais $^{7,11,14,17}$.

Os aspectos clínicos macroscópicos das lesões, mais frequentemente observados nos animais com esporotricose, foram crostas, ulcerações e a presença de pus e sangue. Nos felinos com essa micose, estudados por Madrid et al. ${ }^{11}$, as lesões apresentavam nódulos fistulados que drenavam exsudato 
acastanhado e que eventualmente formavam crostas. Em estudo anterior realizado pelo mesmo grupo de autores, as lesões nos felinos com esporotricose disseminada mostravam predominantemente nódulos com úlceras e $\operatorname{crostas}^{14}$. Quanto à localização das lesões, os animais com esporotricose foram mais frequentemente afetados em sua cabeça, seguido de membros torácicos e pélvicos. Outros autores também referiram o predomínio do acometimento das lesões esporotricóticas na cabeça e nos membros dos felinos domésticos ${ }^{11,14,17}$. A localização das lesões pode refletir as áreas que estão mais expostas durante as brigas ou até mesmo que entram em contato com solo ou plantas $^{5,11}$

Em relação às populações de felinos domésticos, provenientes das diferentes áreas geográficas estudadas, não foram observadas diferenças clínico-epidemiológicas, o que aponta para a existência de um curso uniforme dessa epidemia no Estado. Entretanto, essa diferença era esperada, devido às possíveis características divergentes entre os nichos ambientais e a distribuição do fungo em função da urbanização, do nível socioeconômico, dos aspectos climáticos e demográficos entre as regiões pesquisadas. Observou-se que os fatores aqui pesquisados não influenciaram na infecção pelo Sporothrix e no consequente desenvolvimento das lesões cutâneas nos gatos domésticos assistidos.

A cultura micológica é o padrão-ouro para o diagnóstico da esporotricose, pois somente por meio dela é possível o isolamento do agente causador da doença ${ }^{3}$. Cerca de metade dos animais atendidos tiveram a cultura micológica positiva $(49,5 \%)$, enquanto Schubach et al. ${ }^{5,7}$ obtiveram percentuais maiores (100,0\%). Tal diferença pode ser atribuída ao fato do Instituto Nacional de Infectologia Evandro Chagas (INI) da Fundação Oswaldo Cruz (Fiocruz) representar centro de referência em esporotricose felina na capital do Rio de Janeiro, no qual são assistidos animais previamente diagnosticados pelos médicos veterinários. De fato, a presente pesquisa foi realizada via inclusão de animais por profissionais em diferentes locais de atendimento no Estado, e, portanto, os dados aqui obtidos refletem a rotina clínica de consultórios e clínicas veterinárias. Dessa forma, considera-se o presente desenho de estudo como representativo da rotina diagnóstica da esporotricose felina em áreas endêmicas. Como o esperado, a cultura micológica teve alta sensibilidade, corroborando achados previamente publicados $7,5,11,14$.

Contrariando o senso comum de que o uso de antifúngicos seria decisivo para o insucesso do isolamento do agente em cultura, observou-se, no presente estudo, que, para o expressivo percentual de animais em vigência de tratamento específico, foi possível recuperar fungos do complexo $S$. schenckii a partir das lesões. Alguns fatores podem ter influenciado nesse resultado, como a apresentação do medicamento, a dose prescrita, a possível resistência da cepa ao antifúngico preconizado e, até mesmo, os tratamentos com interrupção devido à fuga, à rejeição do animal à administração do fármaco por via oral e à desistência do proprietário pela dificuldade na administração ou custo do tratamento. Tais fatores poderiam diminuir a eficácia do tratamento, facilitando a proliferação do fungo e sua posterior recuperação ${ }^{18,19}$.

Conforme esperado, a sensibilidade da citopatologia por imprint detectada foi menor $(52,6 \%)$ do que a encontrada na cultura micológica $(95,2 \%)$; mas essa sensibilidade, pela citopatologia, foi maior $(81,6 \%)^{15}$ para um grupo de felinos assistido pelo centro de referência do Rio de Janeiro para o diagnóstico da esporotricose (INI/Fiocruz), validando a técnica como recurso seguro, rápido e de baixo custo para o diagnóstico presuntivo da esporotricose em gatos domésticos ${ }^{15,20}$. Assim como referido para a cultura micológica, os dados aqui obtidos são provenientes de diferentes centros de assistência animal. A confecção da lâmina durante - atendimento clínico e sua análise laboratorial são determinantes para o sucesso da técnica. Quando o material se encontra em excesso (espesso), escasso ou ainda pobremente corado, o resultado pode ser comprometido. Além disso, a baixa carga fúngica nas lesões requer busca criteriosa em todos os campos da lâmina que, por sua vez, exige treinamento e experiência prévios para a identificação fidedigna das leveduras compatíveis com o Sporothrix ${ }^{15}$.

Curiosamente, ao contrário do que foi observado na cultura micológica, o tratamento prévio com antifúngico interfere na positividade da citopatologia por imprint. Neste estudo, foi possível verificar que a dose diária $\geq 100 \mathrm{mg} /$ dia de itraconazol reduz a confiabilidade da citopatologia negativa. Esse achado preocupa, já que, em áreas endêmicas, não é rara a instituição do tratamento anterior à confirmação laboratorial da esporotricose felina ${ }^{18,19,21}$. Atualmente, - livre acesso às redes sociais e sites de buscas, contendo informações referentes ao tratamento e manejo do animal, nem sempre confiáveis, favorece o tratamento empírico antes mesmo da consulta ao médico veterinário.

Importante ressaltar que, segundo a Nota Técnica $n^{\circ} 3 / 2011$ SVS-SESRJ-FIOCRUZ de 201 122, que tornou a esporotricose felina e humana uma doença de notificação compulsória no estado do Rio de Janeiro, - diagnóstico laboratorial baseia-se exclusivamente no isolamento do Sporothrix. Devido ao número elevado de casos refratários ao tratamento ${ }^{19,23,24}$, é imprescindível a realização da cultura para investigação do agente etiológico visando à definição da espécie e, principalmente, de seu perfil de susceptibilidade aos antifúngicos.

\section{CONCLUSÃO}

Os achados obtidos neste estudo mostram que o diagnóstico por cultura micológica e pela citopatologia por imprint produzem resultados discordantes. Logo, há diferenças entre os testes que devem ser consideradas para a conclusão do diagnóstico na rotina clínica veterinária. A dosagem de itraconazol $\geq 100 \mathrm{mg} /$ dia, 
independente da duração do tratamento, reduz a sensibilidade da citopatologia por imprint para o diagnóstico da esporotricose felina. Portanto, tal método deve ser considerado presuntivo e não exclui o isolamento fúngico em cultivo, o padrão-ouro. $\bigcirc$ resultado representa um alerta para o uso dessa ferramenta como recurso único para a confirmação diagnóstica da esporotricose felina na rotina clínica veterinária. Por fim, ainda que, em minoria, resultados falso-negativos pelo método presuntivo adiam medidas preventivas e terapêuticas dessa importante zoonose, com impacto deletério no controle da epidemia.

\section{APOIO FINANCEIRO}

Bolsas de iniciação científica do Conselho Nacional de Desenvolvimento Científico e Tecnológico, Universidade Federal Fluminense (PIBIC-CNPq-UFF). Bolsas de demanda social da Coordenação de
Aperfeiçoamento de Pessoal de Nível Superior (CAPES), nível mestrado.

Projeto Jovem Cientista do Nosso Estado (E-26/103.198/2011) e bolsa de iniciação científica da Fundação de Amparo à Pesquisa do Estado do Rio de Janeiro.

\section{CONFLITOS DE INTERESSE}

Os autores declararam que não houve conflitos de interesse.

\section{CONTRIBUIÇÃO DOS AUTORES}

Todos os autores contribuíram com a idealização do estudo, a análise e a interpretação dos dados e com a redação do manuscrito, aprovando a versão final publicada. Declaram-se responsáveis pelo conteúdo integral do artigo, garantindo sua precisão e integridade.

\section{REFERÊNCIAS}

1 Marimon R, Cano J, Gené J, Sutton DA, Kawasaki M, Guarro J. Sporothrix brasiliensis, S. globosa, and S. mexicana, three new Sporothrix species of clinical interest. J Clin Microbiol. 2007 Oct;45(10):3198-206.

2 Rodrigues AM, Teixeira MM, Hoog GS, Schubach TMP, Pereira SA, Fernandes GF, et al. Phylogenetic analysis reveals a high prevalence of Sporothrix brasiliensis in feline sporotrichosis outbreaks. PLoS Negl Trop Dis. 2013 Jun;7(6):e2281.

3 Barros MBL, Paes RA, Schubach AO. Sporothrix schenckii and sporotrichosis. Clin Microbiol Rev. 2011 Oct;24(4):633-54.

4 Barros MBL, Schubach AO, Schubach TMP, Wanke B, Lambert-Passos SR. An epidemic of sporotrichosis in Rio de Janeiro, Brazil: epidemiological aspects of a series of cases. Epidemiol Infect. 2008 Sep; 136(9): 11 192-6.

5 Schubach TMP, Schubach AO, Reis RS, Cuzzi-Maya T, Blanco TCM, Monteiro DF, et al. Sporothrix schenckii isolated from domestic cats with and without sporotrichosis in Rio de Janeiro, Brazil. Mycopathologia. 2002 Feb; 153(2):83-6.

6 Silva MBT, Costa MMM, Torres CCS, Galhardo MCG, Valle ACF, Magalhães MAFM, et al. Esporotricose urbana: epidemia negligenciada no Rio de Janeiro, Brasil. Cad Saude Publica. 2012 out;28(10):1867-80.

7 Schubach TMP, Schubach A, Okamoto T, Barros $M B L$, Figueiredo FB, Cuzzi $T$, et al. Evaluation of an epidemic of sporotrichosis in cats: 347 cases (1998-2001). J Am Vet Med Assoc. 2004 May;224(10): 1623-9.
8 Souza LL, Nascente PS, Nobre MO, Meinerz ARM, Meireles MCA. Isolation of Sporothrix schenckii from the nails of healthy cats. Braz J Microbiol. 2006 Jul-Sep;37(3):372-4.

9 Barros MBL, Schubach TP, Coll JO, Gremião ID, Wanke B, Schubach A. Esporotricose: a evolução e os desafios de uma epidemia. Rev Panam Salud Publica. 2010;27(6):455-60.

10 Barros MBL, Schubach TMP, Galhardo MCG, Schubach AO, Monteiro PCF, Reis RS, et al. Sporotrichosis: an emergent zoonosis in Rio de Janeiro. Mem Inst Oswaldo Cruz. 2001 Aug;96(6):777-9.

11 Madrid IM, Mattei AS, Fernandes CG, Nobre MO, Meireles MCA. Epidemiological findings and laboratory evaluation of sporotrichosis: a description of 103 cases in cats and dogs in southern Brazil. Mycopathologia. 2012 Apr; 173(4):265-73.

12 Sanchotene KO, Madrid IM, Klafke GB, Bergamashi M, Terra PPD, Rodrigues AM, et al. Sporothrix brasiliensis outbreaks and the rapid emergence of feline sporotrichosis. Mycoses. 2015 Nov;58(1 1):652-8.

13 Mattei AS, Madrid IM, Santin R, Silva FV, Carapeto LP, Meireles MCA. Sporothrix schenckii in a hospital and home environment in the city of Pelotas/RS - Brazil. An Acad Bras Cienc. 2011 Dec;83(4):1359-62.

14 Madrid IM, Mattei A, Martins A, Nobre M, Meireles $M$. Feline sporotrichosis in the southern region of Rio Grande do Sul, Brazil: clinical, zoonotic and therapeutic aspects. Zoonoses Public Health. 2010 Mar;57(2):151-4. 
15 Silva JN, Passos SRL, Menezes RC, Gremião IDF, Schubach TMP, Oliveira JC, et al. Diagnostic accuracy assessment of cytopathological examination of feline sporotrichosis. Med Mycol. 2015 Nov;53(8):880-4.

16 Gurr E. Synthetic dyes in biology, medicine and chemistry. New York: Academic Press; 1971.

17 Leme LRP, Schubach TMP, Santos IB, Figueiredo FB, Pereira SA, Reis RS, et al. Mycological evaluation of bronchoalveolar lavage in cats with respiratory signs from Rio de Janeiro, Brazil. Mycoses. 2007 May;50(3):210-4.

18 Pereira SA, Schubach TMP, Gremião IDF, Silva DT, Figueiredo FB, Assis NV, et al. Aspectos terapêuticos da esporotricose felina. Acta Sci Vet. $2009 ; 37(4): 331-21$.

19 Chaves AR, Campos MP, Barros MBL, Carmo CN, Gremião IDF, Pereira SA, et al. Treatment abandonment in feline sporotrichosis - study of 147 cases. Zoonoses Public Health. 2013 Mar;60(2): 149-53.

20 Pereira SA, Menezes RC, Gremião IDF, Silva JN, Honse CO, Figueiredo FB, et al. Sensitivity of cytopathological examination in the diagnosis of feline sporotrichosis. J Feline Med Surg. 2011 Apr; 13(4):220-3
21 Gremião IDF, Pereira SA, Rodrigues AM, Figueiredo FB, Nascimento Júnior A, Santos IB, et al. Tratamento cirúrgico associado à terapia antifúngica convencional na esporotricose felina. Acta Sci Vet. 2006;34(2):221-3.

22 Secretaria de Estado de Saúde do Rio de Janeiro. Subsecretaria de Vigilância em Saúde. Superintendência de Vigilância Epidemiológica e Ambiental. Coordenação de Vigilância Epidemiológica. Divisão de Transmissíveis e Imunopreviníveis. Nota técnica n³/2011-GDTVZ/ DTI/CVE/SVEA/SVS-SESRJ e IPEC/FIOCRUZ, de 5 de outubro de 2011: orientações sobre vigilância da esporotricose no Estado do Rio de Janeiro. Rio de Janeiro: Secretaria de Estado de Saúde, Instituto de Pesquisa Clínica Evandro Chagas/Fiocruz; 2011.

23 Gremião IDF, Schubach TMP, Pereira SA, Rodrigues AM, Chaves AR, Barros MBL. Intralesional amphotericin $B$ in a cat with refractory localised sporotrichosis. J Feline Med Surg. 2009 Aug; 11 (8):720-3.

24 Gremião IDF, Miranda LHM, Reis EG, Rodrigues AM, Pereira SA. Zoonotic epidemic of sporotrichosis: cat to human transmission. PLoS Pathog. 2017 Jan;13(1):e1006077.

Recebido em / Received: 13/3/2017 Aceito em / Accepted: 11/12/2017 